\title{
Angular distribution of diffuse reflectance in biological tissue
}

\author{
Jinjun Xia and Gang Yao* \\ Department of Biological Engineering, University of Missouri-Columbia, Columbia, Missouri 65211, USA \\ *Corresponding author: yaog@missouri.edu \\ Received 16 April 2007; revised 4 June 2007; accepted 21 June 2007; \\ posted 5 July 2007 (Doc. ID 82113); published 5 September 2007
}

\begin{abstract}
We measured angular-resolved diffuse reflectance in tissue samples of different anisotropic characteristics. Experimental measurements were compared with theoretical results based on the diffusion approximation. The results indicated that the angular distribution in isotropic tissue was the same as in isotropic phantoms. Under normal incidence, the measured angular profiles of diffuse reflectance approached the Lambertian distribution when the evaluation location was far away from the incident point. The skewed angular profiles observed under oblique incidence could be explained using the diffuse model. The anisotropic tissue structures in muscle showed clear effects on the measurements especially at locations close to the light incidence. However, when measuring across the muscle fiber orientations, the results were in good agreement with those obtained in isotropic samples. (C 2007 Optical Society of America

OCIS codes: $\quad 170.3660,170.7050,170.6930,290.1350,290.1990$.
\end{abstract}

\section{Introduction}

Photon migration methods have been widely applied in biomedical fields for in vivo early disease diagnosis [1-4]. In many applications, optical fibers are used to deliver and receive light because they are flexible and can be easily incorporated into endoscopes. Since a fiber optic probe has a limited acceptance angle, its geometrical design [5], and the spatial and angular distribution [6,7] of the light signals may affect the amount of light that is detected, and need to be characterized. In addition, the angular profile of diffuse reflectance from the tissue surface is an important factor for the realistic rendering of biological tissues in computer graphics [8]. In such applications, the tissue surface is usually assumed to be a Lambertian source under the illumination of an external light source where the surface luminous intensity is proportional to the cosine of the angle formed between the surface normal direction and the view direction. These aforementioned applications can certainly benefit from a detailed study to determine the actual

0003-6935/07/266552-09\$15.00/0

(C) 2007 Optical Society of America angular profile of diffuse reflectance in biological tissues.

Kienle et al. [9] studied the angular distribution of diffuse reflectance under normal incidence of a pencil beam using a Monte Carlo simulation. Their results showed that the distribution was very close to the Lambertian distribution except for locations proximate to the incident point. In a later study, $\mathrm{Qu}$ et al. [6] also found that when they used Monte Carlo simulation, the angular profile of diffuse reflectance was almost Lambertian under a normal incidence of a broad beam. However, under oblique incidence, the angular distribution was different from the Lambertian distribution. Recently, Gebhart et al. [7] conducted comprehensive studies on this topic using phantoms and simulations, and investigated the effects of sample optical properties.

In this study, we further studied the angular profile of diffuse reflectance from turbid media. Specifically, we measured the angular distribution of diffuse reflectance in real biological tissues, and compared the results with those obtained from tissue equivalent phantoms. In addition, we found that a diffuse model of light transport can be applied to explain the skewing effects $[6,7]$ observed under oblique incidence. We conducted measurements in two different kinds of bi- 
ological tissues: isotropic and anisotropic. Isotropic tissues have no specific structural orientations. Although tissues are commonly considered to be isotropic in practical applications, many biological tissues are inherently anisotropic [10-15]. These anisotropic samples have well organized structural orientations so that photons scattering within the medium depends on the incident direction. Such global anisotropy is conceptually different from single scattering anisotropy, which is determined by scattering phase function. Using skeletal muscles [15] as models, we showed that the angular profiles of diffuse reflectance depend on the measurement orientations in such anisotropic tissues, and may have large deviations from the Lambertian distribution.

\section{Materials and Methods}

\section{A. Samples}

Tissue equivalent phantoms were made from gelatin type B (CAS 9000-70-8, Thermo Fisher Scientific Inc., Waltham, MA, USA), and 20\% intralipid (CAS 6889065-3, Sigma-Aldrich, Inc., St. Louis, MO, USA). Various concentrations of intralipid were used to make samples of different reduced scattering coefficients $\left(\mu_{s}{ }^{\prime}\right)$. Chicken liver tissues were acquired from a local grocery store to represent isotropic tissues. Striated muscle samples (Longissimus dorsi) were acquired from the Meat Science Laboratory at the University of Missouri-Columbia to represent anisotropic tissues.

\section{B. Experimental Setup}

The experimental setup is illustrated in Fig. 1. A coordinate was set so that the origin was coincident with the incident point at the sample surface, and the $z$-axis pointed into the sample. Two optical fibers with $400 \mu \mathrm{m}$ core diameters, and 0.22 N.A. were used. One fiber was connected to a LED light source $(692.4 \mathrm{~nm})$, and was used to deliver the light to the

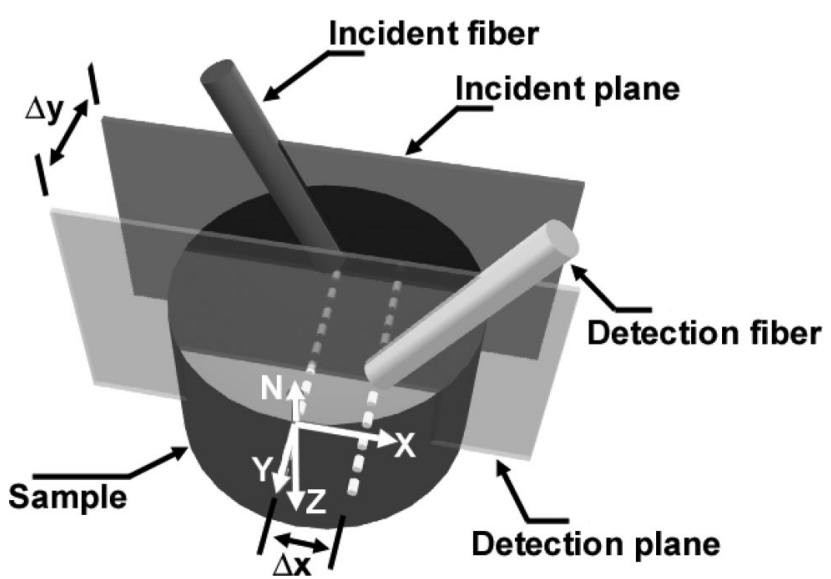

Fig. 1. Schematic diagram for measuring the angular resolved diffuse reflectance. In the experiments, the incident fiber delivered light at $0^{\circ}$ or $40^{\circ}$ within the incident plane; the detection fiber collected reflectance while rotating within the detection plane. sample surface; the other fiber was used to collect diffuse reflectance.

Each fiber tip was attached to a manual rotational stage for adjusting their angular positions. The two rotational stages were mounted systematically so that the two fiber tips rotated within two parallel planes: the incident plane and the detection plane. The two planes were extended along the $x$-axis in Fig. 1. The incident plane was defined by the incident fiber and the normal direction of the sample surface (N). The detection plane was defined by the detection fiber and the normal direction on sample surface. The two rotational axes (along the $y$-axis) were perpendicular to these two planes, and they were precisely calibrated so that they were located at the sample surface.

The detection fiber setup was mounted on a two dimensional translation stage so that the fiber tip can be translated laterally and longitudinally within the measurement window. The longitudinal movement of the detection fiber changed the distance between the incident plane and the detection plane $(\Delta y)$, while the lateral movement changed the detection position within the same detection plane. The angular resolved reflectance was measured by rotating the detection fiber tip to different angles to collect diffuse reflectance. The measured angles were from $-40^{\circ}$ to $40^{\circ}$ corresponding to the normal direction $(\mathbf{N})$. The incident fiber was adjusted either perpendicular to the sample surface or at an oblique degree $40^{\circ}$ to the normal direction.

To ensure a smooth measurement surface, tissue samples were mounted beneath a thin cover glass $(n=1.52)$ with thickness of $0.13-0.16 \mathrm{~mm}$. Although its refractive index was considered in a diffuse calculation, the refraction and reflection at the top glassair interface may change the angular distribution and may contribute to the slight difference observed in this study between the experiment and the calculation. Using our coupling system, the divergence angle of the light beam exiting from the incident fiber was measured as $6.5^{\circ}$ (half angle). The fibers were located $1.0 \mathrm{~mm}$ above the thin cover glass. The beam diameter at the sample surface was estimated to be $\sim 0.63 \mathrm{~mm}$ at normal incidence. When positioned at normal $\left(0^{\circ}\right)$ to the sample surface, the collection fiber received light from an area of $0.85 \mathrm{~mm}$ in diameter from the sample surface. To compensate a larger collection area at other angles, the measured light intensity was multiplied by the cosine of the detection angle.

\section{A Diffuse Model of the Angular Distribution of Diffuse Reflectance}

Photon migration in turbid media is commonly described with radiative transfer theory $[16,17]$. Theoretical results based on this theory are in good agreement with experimental observations in turbid media $[18,19]$. For many applications is highly scattering media, the diffusion approximation can be applied to simplify the radiative transfer theory. The solution of diffusion equation in a semi-infinite scat- 
tering medium $[20,21]$ was obtained more than a decade ago and has been widely used since then. Based this solution, the fluence rate $\phi(\mathbf{r})$ of a pencil beam incident perpendicularly upon a semi-infinite scattering medium can be obtained using a dipole source with an extrapolated boundary condition. Specifically, the positive point source is located one transport mean-free-path $\left(\mathrm{mfp}^{\prime}\right)$ beneath the incident point, where $1 \mathrm{mfp}^{\prime}=1 / \mu_{t}{ }^{\prime}$ and $\mu_{t}{ }^{\prime}=\mu_{s}{ }^{\prime}+\mu_{a}$. A negative point source is located $1 / \mu_{t}{ }^{\prime}+4 A D$ above the incident point, where $D$ is the diffuse coefficient and $A$ is the coefficient accounting for the surface reflection [21]. The spatially resolved diffuse reflectance $R(r)$ along the surface normal direction was derived as

$$
R(r)=-\left.D \frac{\partial \phi}{\partial z}\right|_{z=0},
$$

where $D$ is the diffuse coefficient, $r$ is the distance between the evaluation location and the incident location, and $z$ is the axis aligned with the normal vector $\mathbf{N}$ in Fig. 1 (but it is pointing into the sample), and $z=0$ indicates the sample surface. The diffuse reflectance at the sample surface along an arbitrary direction can be calculated as

$$
R(r, \mathbf{u})=-\left.D\left(\frac{\partial \phi}{\partial x} u_{x}+\frac{\partial \phi}{\partial y} u_{y}+\frac{\partial \phi}{\partial z} u_{z}\right)\right|_{z=0},
$$

where $\mathbf{u}=\left(u_{x}, u_{y}, u_{z}\right)$ are the directional angles (cosine) along the three axes, and $u_{x}^{2}+u_{y}^{2}+u_{z}^{2}=1$. At normal incidence, if the angular profile is measured in such a way that the rotational axis passes through the origin, Eq. (2) predicts a Lambertian distribution. For example, without losing the generality, if the detection fiber rotates within the detection plane and around the $y$-axis (Fig. $1, \Delta x=0, u_{y}=0$ ), Eq. (2) becomes

$$
R\left(r, \theta_{z}\right)=-\left.D \frac{\partial \phi}{\partial z}\right|_{z, x=0} \cos \left(\theta_{z}\right) \propto \cos \left(\theta_{z}\right),
$$

where $\theta_{z}$ is the detection angle formed with the $z$-axis. From Eq. (2), if the rotation axis does not pass through the incident point, the angular distribution is not Lambertian.

If the light beam is incident at an oblique angle of $\theta_{z}$ within the incident plane shown in Fig. 1, the positive point source (the diffuse center) of the dipole system is shifted by a distance of $\Delta x[22,23]$ along the $x$-axis:

$$
\Delta x=\frac{\sin \theta_{t}}{\mu_{t}^{\prime}},
$$

where $\theta_{t}$ is the refraction angle inside the medium. Therefore the angular distribution of the diffuse reflectance is still Lambertian when the measurement axis passes through the diffuse center. However, if the measurement axis passes the incidence point, the obtained angular profile is no longer Lambertian, and can be calculated using Eq. (2).

Due to the nature of diffuse approximation, the angular resolved diffuse reflectance that is calculated with the above methods is not valid when the evaluation site is close to the light incident location. To investigate this type of effect, we conducted measurements at different distances between the incident plane and the detection plane (Fig. 1): from close $\left(\Delta y<1 \mathrm{mfp}^{\prime}\right)$ to far away $\left(\Delta y>2 \mathrm{mfp}^{\prime}\right)$. All measurement distances were measured in units of the transport mean-free-path to account for tissues with different optical properties. We used the oblique incidence reflectometry [24-27] method to measure the sample absorption coefficients $\left(\mu_{a}\right)$ and reduced scattering coefficients $\left(\mu_{s}{ }^{\prime}\right)$. In the measurements, the tissue refractive index was assumed to be 1.37 . The accuracy of the measurement system was $10 \%$ using calibrated scattering phantoms [27].

\section{Results and Discussions}

\section{A. Normal Incidence}

The angular distributions of diffuse reflectance from isotropic intralipid phantoms were measured when the delivery fiber was positioned perpendicularly to the sample surface. The absorption coefficients of these phantoms were very small compared to the scattering coefficients. The rotational axis for the measurements was on the line that passed through the incident point ( $\Delta x=0$ in Fig. 1), and was perpendicular to both the incident plane and the detection plane. The measured angular distributions were normalized to the value measured at $0^{\circ}$. The normalized angular distributions of diffuse reflectance were shown in Fig. 2 for $\Delta y=0.8$ and $2.0 \mathrm{mfp}^{\prime}$. The data points shown were averaged over several measurements. The standard deviations were small and may not show up in the graphs as error bars. It can be seen that the angular profiles were all symmetric centering at the incident point. Samples with different reduced scattering coefficients were measured in the experiments. However, when the distance was measured in the unit of $\mathrm{mfp}^{\prime}$, the reduced scattering coefficient had no influence on the angular distribution of diffuse reflectance disregarding the measurement distances. As we show in Figs. 2(a) and 2(b), the difference among the three phantoms was less than $1.6 \%$.

Since the measurement was centered at the axis passing through the origin, the diffuse theory predicted a Lambertian profile of the angular distribution as we describe in Eq. (3). Figure 2(c) shows the measured angular profiles at three locations of different distances away from the incident point (the $\Delta y$ in Fig. 1): 0.8, 2.0, and $3.64 \mathrm{mfp}^{\prime}$. The $\mu_{s}^{\prime}$ of the phantom was $5.8 \mathrm{~cm}^{-1}$, and the absorption coefficient was negligible. The deviation was usually greater at larger detection angles. It can be seen that at larger distances, the angular profile approached the Lambertian distribution. As we expected, there were larger deviations when the evaluation point was close 

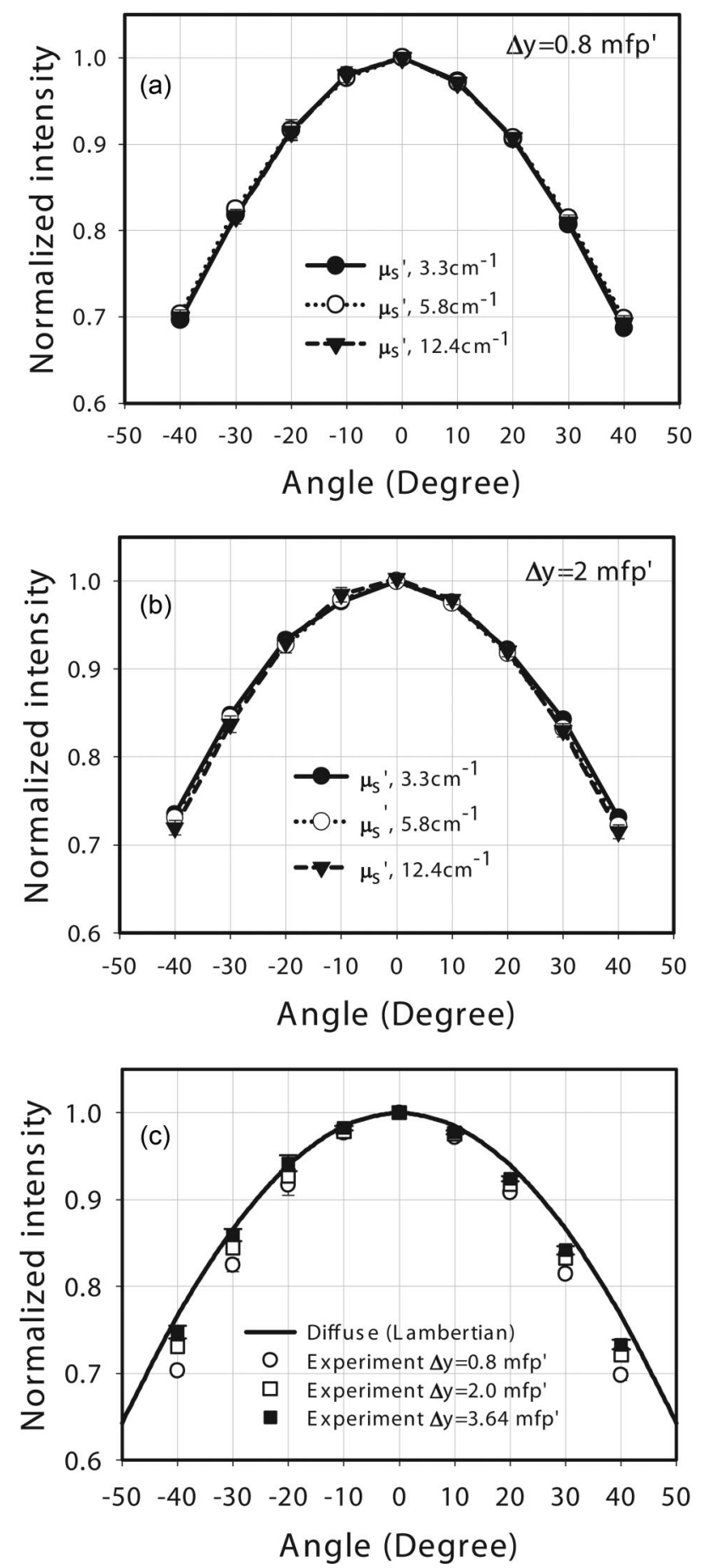

Fig. 2. Normalized-angular distribution of diffuse reflectance for three phantoms at (a) $\Delta y=0.8 \mathrm{mfp}^{\prime}$ and (b) $\Delta y=2.0 \mathrm{mfp}^{\prime}$. Optical properties of the phantoms were: $\mu_{s}^{\prime}=3.3 \mathrm{~cm}^{-1}$ and $\mu_{a}$ $=2.0 \times 10^{-4} \mathrm{~cm}^{-1}, \mu_{s}^{\prime}=5.8 \mathrm{~cm}^{-1}$ and $\mu_{a}=1.0 \times 10^{-4} \mathrm{~cm}^{-1}$, and $\mu_{s}{ }^{\prime}=12.4 \mathrm{~cm}^{-1}$ and $\mu_{a}=1.0 \times 10^{-4} \mathrm{~cm}^{-1}$. The comparison between experiments and the Lambertian distribution was shown in (c), where the phantom that we used had $\mu_{s}{ }^{\prime}=5.8 \mathrm{~cm}^{-1}$ and $\mu_{a}=$ $1.0 \times 10^{-4} \mathrm{~cm}^{-1}$.

to the incident point. The maximal differences from the Lambertian distribution were $8.9 \%, 5.8 \%$, and $4.3 \%$ at $\Delta y=0.8,2.0$, and $3.64 \mathrm{mfp}^{\prime}$ distances, respectively.

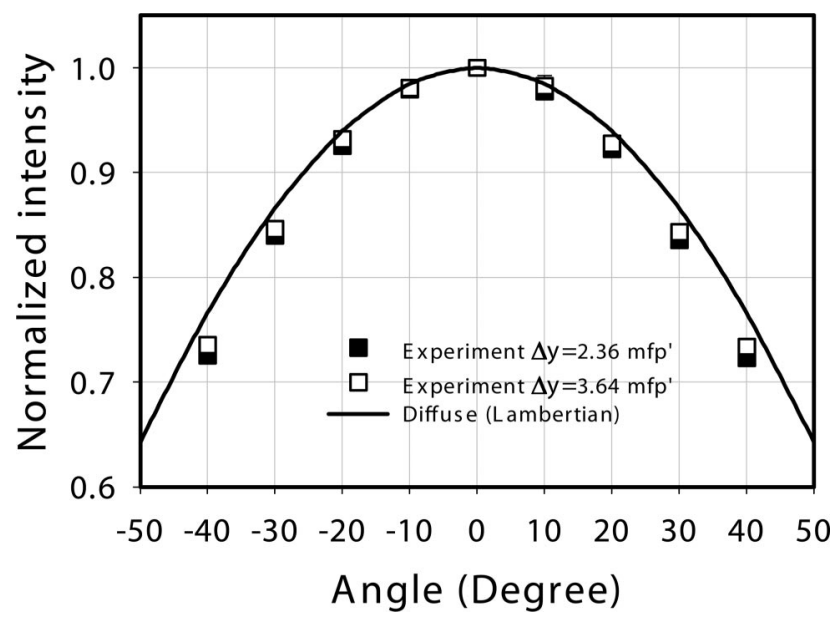

Fig. 3. Normalized angular distribution of diffuse reflectance measured in chicken liver tissue at $\Delta y=2.36$ and $\Delta y=3.64 \mathrm{mfp}^{\prime}$ from the incident location. (Sample optical properties were $\mu_{s}{ }^{\prime}=8.7$ $\mathrm{cm}^{-1}$ and $\mu_{a}=0.5 \mathrm{~cm}^{-1}$.)

In the chicken liver tissue, the normalized angular distributions of diffuse reflectance measured at $\Delta y$ $=2.36$ and $3.64 \mathrm{mfp}^{\prime}$ from the incidence were shown in Fig. 3. The optical properties of the tissue were $\mu_{s}{ }^{\prime}=8.7 \mathrm{~cm}^{-1}$ and $\mu_{a}=0.5 \mathrm{~cm}^{-1}$. Because of the large reduced scattering coefficient of the liver tissue, the minimum distance to the incident point that we could measure was $\Delta y=2.36 \mathrm{mfp}^{\prime}$ to avoid the collision of the delivery fiber with the detection fiber. Figure 3 indicated that the angular distributions of diffuse reflectance were almost identical as in Intralipid phantoms even though the liver had much greater absorption than the Intralipid phantoms. As in phantoms, the angular profile was slightly underestimated compared with the Lambertian distribution. The maximal differences from the Lambertian distribution were $5.6 \%$ and $4.2 \%$ at $\Delta y=2.36$ and $3.64 \mathrm{mfp}^{\prime}$ distances, respectively.

Light propagation in skeletal muscles is not isotropic because of the muscle fibers [28] and the periodic sarcomere structures $[15,27]$. Because previous studies indicated that optical properties of muscle depended on the measurement orientation [29], we chose three different geometrical arrangements in our measurements. In the first arrangement (the "cross" arrangement), the detection plane was perpendicular to the muscle fiber orientation, i.e., muscle fibers were aligned with the $y$-axis in Fig. 1 . In the second arrangement (the "parallel" arrangement), the detection plane was parallel to the muscle fibers, i.e., muscle fibers were aligned with the $x$-axis in Fig. 1 . In the third arrangement (the "end" arrangement), the cross section of the muscle bundle was used as the measurement surface, i.e., the muscle fibers were aligned with the $z$-axis in Fig. 1.

All results with the aforementioned geometrical arrangements in skeletal muscle were shown in Fig. 4 along with the Lambertian distribution predicted from diffuse theory. In the cross arrangement, at three distances from $\Delta y=0.8-3.64 \mathrm{mfp}^{\prime}$, the mea- 

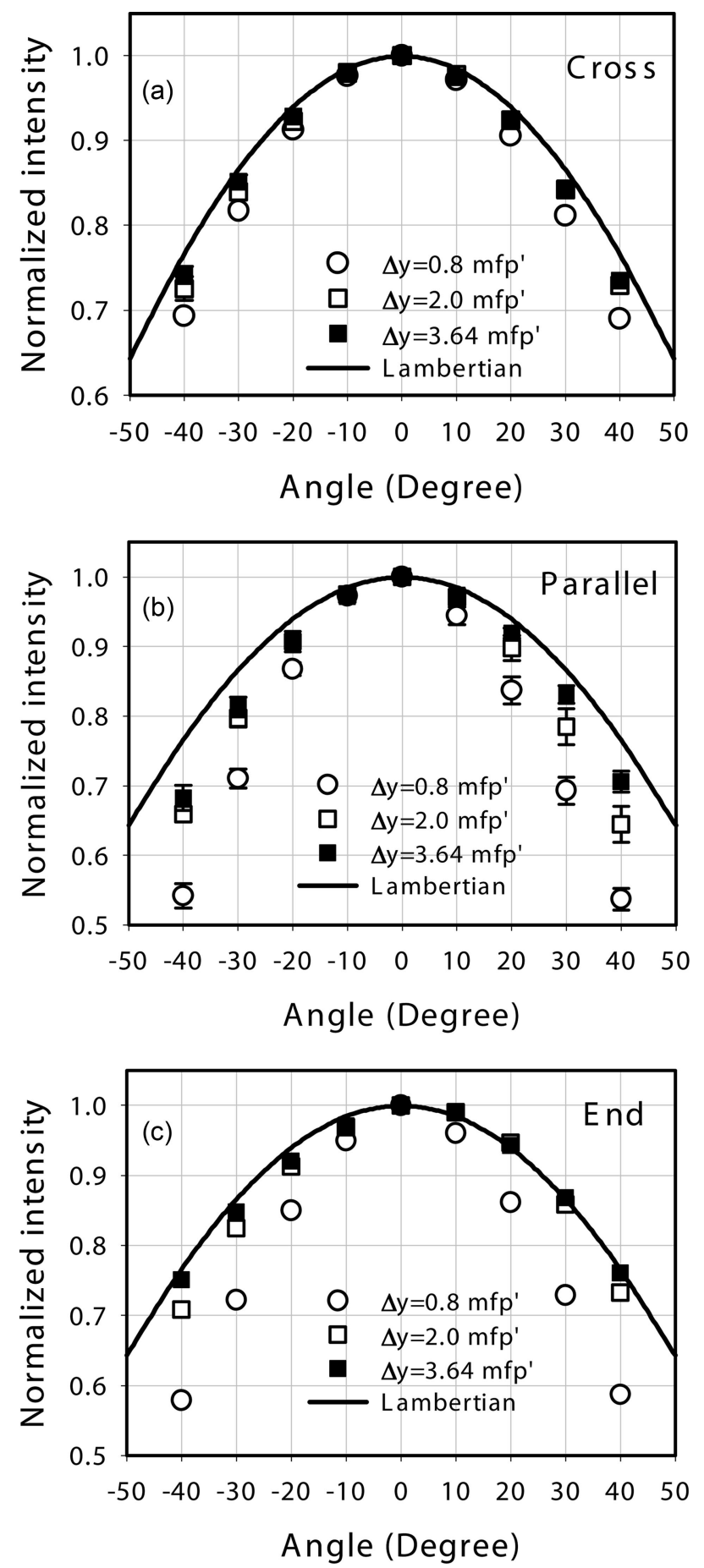

Fig. 4. Normalized angular distribution of diffuse reflectance in muscle measured with (a) across, (b) parallel, and (c) end arrangements. Optical properties of muscle tissue were: $\mu_{s}{ }^{\prime}=3.6 \mathrm{~cm}^{-1}$ and $\mu_{a}=2.0 \times 10^{-2} \mathrm{~cm}^{-1}$ measured with the cross arrangement, $\mu_{s}{ }^{\prime}$ $=6.0 \mathrm{~cm}^{-1}$ and $\mu_{a}=7.5 \times 10^{-2} \mathrm{~cm}^{-1}$ measured with the parallel arrangement, and $\mu_{s}^{\prime}=4.6 \mathrm{~cm}^{-1}, \mu_{a}=4.2 \times 10^{-3} \mathrm{~cm}^{-1}$ measured with end arrangement.

sured angular distributions were all nearly identical to the corresponding distributions in phantoms. The maximal relative errors from the ideal Lambertian distribution were $9.9 \%, 5.3 \%$, and $4.1 \%$ at $\Delta y=0.8$, 2.0 , and $3.64 \mathrm{mfp}^{\prime}$ distances, respectively. This indi- cated the similarity between this geometrical arrangement in skeletal muscle tissue and the isotropic tissue phantom. In the parallel arrangement, at a close distance, the measured angular distribution of diffuse reflectance was much narrower than the distribution in the phantom. However, the discrepancy became smaller as the distance increased with relative errors from the Lambertian distribution changing from $29.9 \%$ at $\Delta y=0.8 \mathrm{mfp}^{\prime}$ to $10.9 \%$ at $3.64 \mathrm{mfp}^{\prime}$. Similarly, in the end arrangement, at a close distance, its angular distribution was also significantly narrower than the correspondents in the phantom tissue. And it became closer to the result obtained in the phantom as distance increased. The maximal relative errors from the ideal Lambertian distribution were $24.5 \%, 7.5 \%$, and $2.0 \%$ at $\Delta y$ $=0.8,2.0$, and $3.64 \mathrm{mfp}^{\prime}$ distances, respectively. For both the cross and end arrangements, the angular profiles of diffuse reflectance approached the Lambertian distribution when the evaluation location $(\Delta y)$ was greater than $2 \mathrm{mfp}^{\prime}$.

The above results indicated that under the normal incidence, the angular distributions of diffuse reflectance in an isotropic tissue (live tissue) were the same as those measured in tissue phantoms. In anisotropic tissues such as skeletal muscle, the results measured across the muscle fibers had little difference with those isotropic tissue or phantoms. However, in the other two geometrical arrangements, i.e., the parallel and end measurements, the results were quite different from those obtained in isotropic media, especially at small measurement distances. For all measurements except for the parallel arrangement in muscle, the angular profiles were very close to the Lambertian distribution at larger distances $\left(\Delta y>1 \mathrm{mfp}^{\prime}\right)$. Nevertheless, the anisotropic tissue structures did affect the angular distribution of diffuse reflectance as we show in the muscle results.

\section{B. Oblique Incidence}

In this study, the light was incident upon the sample at $40^{\circ}$ to the normal direction of the surface. At oblique incidence, two measurement locations are particularly interesting: the incident point and the diffuse center. The diffuse center deviates from the incident point as shown in Eq. (4). For each sample, we measured the angular resolved reflectance centered on two different axes. One axis passed through the incident point (the "incident line") and the other line passed through the diffuse center (the "diffuse line"). Both lines were perpendicular to both the incident and the detection planes. The diffuse center can be calculated using Eq. (4) after the sample optical properties were measured. In addition, we also used the symmetrical center of the diffuse reflectance as a comparison by matching the reflectance signal measured on both sides of the incident point. For all the measurements, the differences in diffuse centers obtained by these two methods were less than $4 \%$.

Figure 5(a) shows the results obtained in Intralipid phantoms at different measurement locations along the incident line $(\Delta x=0)$. Other than the symmetric 

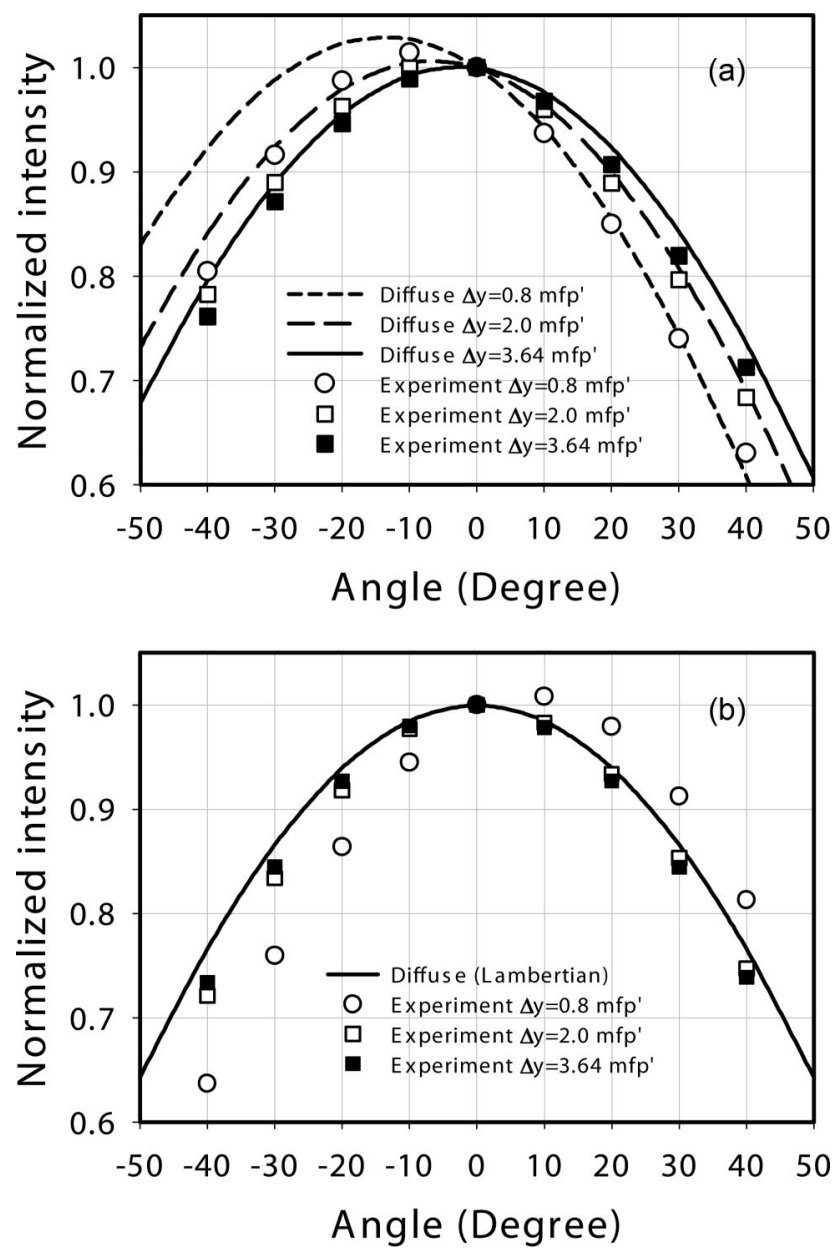

Fig. 5. Normalized angular distribution of diffuse reflectance of intralipid phantom under $40^{\circ}$ incidence measured around an axis passing through (a) incident point, and (b) diffuse center. The optical properties of the phantom were the same as in Fig. 2(c).

profiles observed in the normal incidence situation (Fig. 2), the angular distributions were all skewed even at large distances where photons have been diffused. The skewing was also calculated using Eq. (2). It can be seen from Fig. 5(a) that the calculated curves had better agreement with the experimental data at larger distances. The maximal relative errors between experiment and calculation were $35.0 \%$, $6.9 \%$, and $4.3 \%$ at $\Delta y=0.8,2.0$, and $3.64 \mathrm{mfp}^{\prime}$ distances, respectively. The optical properties of the phantom used in Fig. 5 were the same as in Fig. 2(c). We conducted measurements in phantoms of different scattering coefficients, and found no significant difference in angular distributions. As in normal incidence, this suggested that optical properties did not affect the angular distribution of diffuse reflectance when $\mathrm{mfp}^{\prime}$ was used as the distance measure.

Based on diffusion approximation, when the detection fiber was aimed toward the diffuse center, it collected more light than when aiming it away from the diffuse center. If the angular resolved reflectance was measured at the diffuse line, the measured angular distribution should be the same as that mea- sured at the incident line under normal incidence. Figure 5(b) shows the results obtained in intralipid phantoms along the diffuse line. The maximal relative errors between experiment and calculation were $16.8 \%, 5.9 \%$, and $4.2 \%$ at $\Delta y=0.8,2.0$, and $3.64 \mathrm{mfp}^{\prime}$ distances, respectively. At large distances of $\Delta y=2$ and $3.64 \mathrm{mfp}^{\prime}$, the distributions were nearly symmetrical and close to Lambertian distribution. However, at close distance $\left(\Delta y=0.8 \mathrm{mfp}^{\prime}\right)$, the angular distribution was still skewed. Such a discrepancy was partially attributed to the ballistic photons received at this small detector-source distance. In addition, this type of deviation can also be caused by small errors in aligning the detection fiber with the diffuse center. We found that the distribution measured at $\Delta y=$ $0.8 \mathrm{mfp}^{\prime}$ was sensitive to sample optical properties. We noticed that, especially for samples of large reduced scattering coefficients, the distance between the incidence and diffuse centers was small, and any small errors can have big a influence.

In liver tissue, when the measurement was centered at the incident line $(\Delta x=0$ in Fig. 1), the measured angular distribution was the same as in the phantoms [Figs. 6(a) and 5(a)]. Measurements were conducted at two locations of $\Delta y=2.36$ and $3.64 \mathrm{mfp}^{\prime}$ from the incident point ( $\Delta y$ in Fig. 1). The angular distributions at both distances were skewed. The experimental measurements had good agreement with diffuse calculations at larger distances. The maximal relative error between experiment and calculation was $4.7 \%$ at $\Delta y=3.64 \mathrm{mfp}^{\prime}$. The angular distributions measured at the diffuse line at $\Delta y$ $=2.36$ and $3.64 \mathrm{mfp}^{\prime}$ from incidence were shown in Fig. 6(b). It can be seen that the results were similar to those measured in the phantoms. The angular profiles became symmetrical and matched the Lambertian distribution. The maximal relative errors between experiment and calculation were $5.9 \%$ and $4.2 \%$ at $\Delta y=2.36$ and $3.64 \mathrm{mfp}^{\prime}$ distances, respectively.

For all three geometrical arrangements in the skeletal muscle, the angular distributions of diffuse reflectance at the incident line were skewed (Fig. 7). When measured across the fibers, the angular distributions at all three distances, no matter how near or far, were the same as in the solid phantoms, which were the same as in normal incidence. When the detection plane was parallel to the muscle fiber direction (the parallel arrangement) or at the cross section of the fibers (the end arrangement), the results were different from the phantoms, especially at a closer distance to the incident point.

The results calculated from the diffuse theory [Eq. (2)] were also shown in Fig. 7. It can be seen that, in the cross configuration, the diffuse model was in good agreement with our experimental observation. Quantitatively, the maximal relative errors between experiment and calculation were $10.7 \%, 3.7 \%$, and $3.6 \%$ at $\Delta y=0.8,2.0$, and $3.64 \mathrm{mfp}^{\prime}$ distances, respectively. For the parallel and end configurations, there were larger discrepancies between theory and experiments although the diffuse theory depicted a correct 

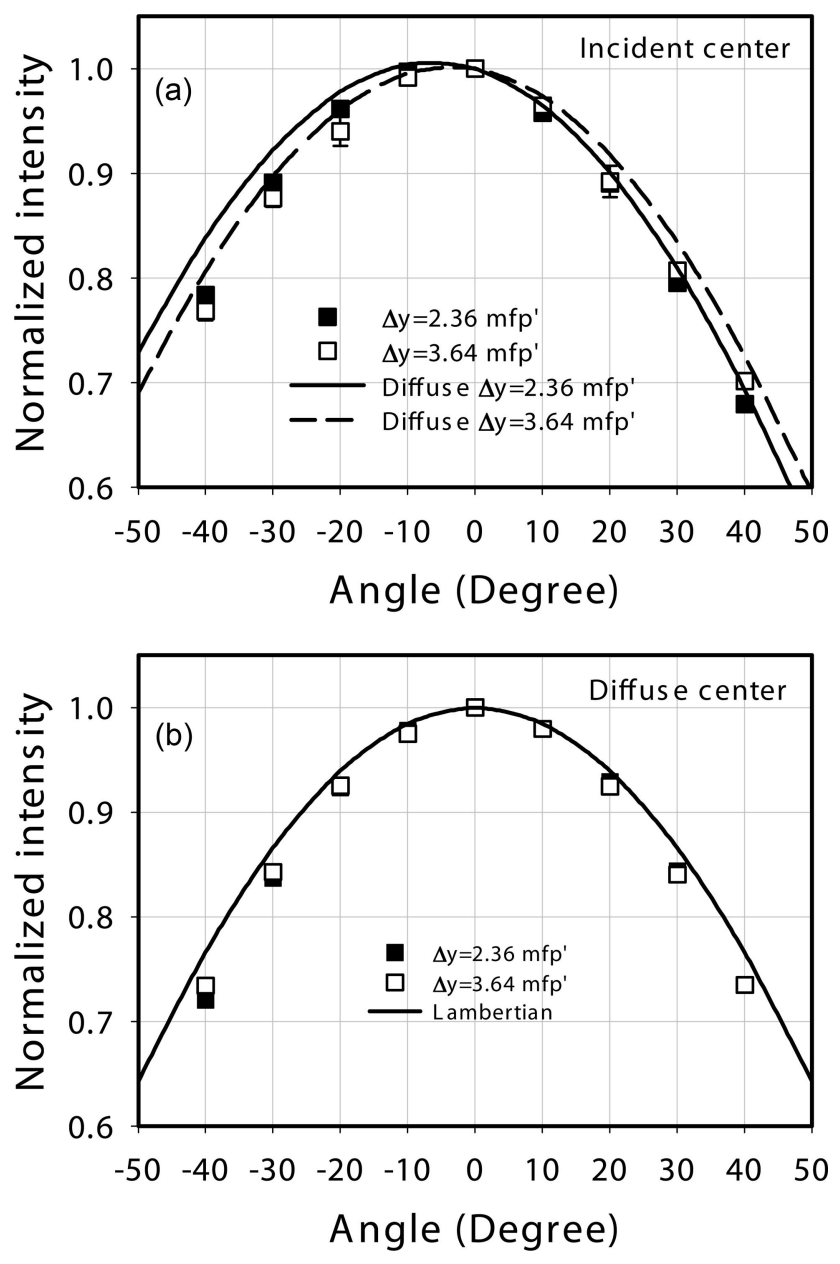

Fig. 6. The normalized angular distribution of diffuse reflectance centered at the incident line in liver tissue under $40^{\circ}$ incidence. The distance between the incident and detection planes was $\Delta y$ $=2.36$ and $\Delta y=3.64 \mathrm{mfp}^{\prime}$ (Sample optical properties were $\mu_{s}{ }^{\prime}$ $=8.7 \mathrm{~cm}^{-1}$ and $\mu_{a}=0.5 \mathrm{~cm}^{-1}$.).

trend. Even at a larger distance of $\Delta y=3.64 \mathrm{mfp}^{\prime}$, the errors between experiment and calculation were still as large as $7.5 \%$ and $11.7 \%$ for parallel and end arrangements, respectively. However, at the small distance of $\Delta y=0.8 \mathrm{mfp}^{\prime}$, all three geometrical arrangements produced different results from the diffuse theory because the diffuse theory cannot be applied at close proximities to the incident location.

Figure 8 shows the results in muscle measured at the diffuse line. The angular profiles became symmetrical as was predicted from the diffuse theory for all the three different measurement arrangements, except for the ones measured at a small distance $(\Delta y$ $\left.=0.8 \mathrm{mfp}^{\prime}\right)$. The cross arrangement showed the best match with the Lambertian distribution at larger distances. At $\Delta y=3.64 \mathrm{mfp}^{\prime}$, the maximal relative error between experiment and calculation was $4.1 \%$. On the other hand, the parallel and end arrangements still deviated from the Lambertian distribution even at larger measurement distances. At $\Delta y=3.64 \mathrm{mfp}^{\prime}$, the errors between experiment and calculation were still as large as $8.7 \%$ and $11.3 \%$ for
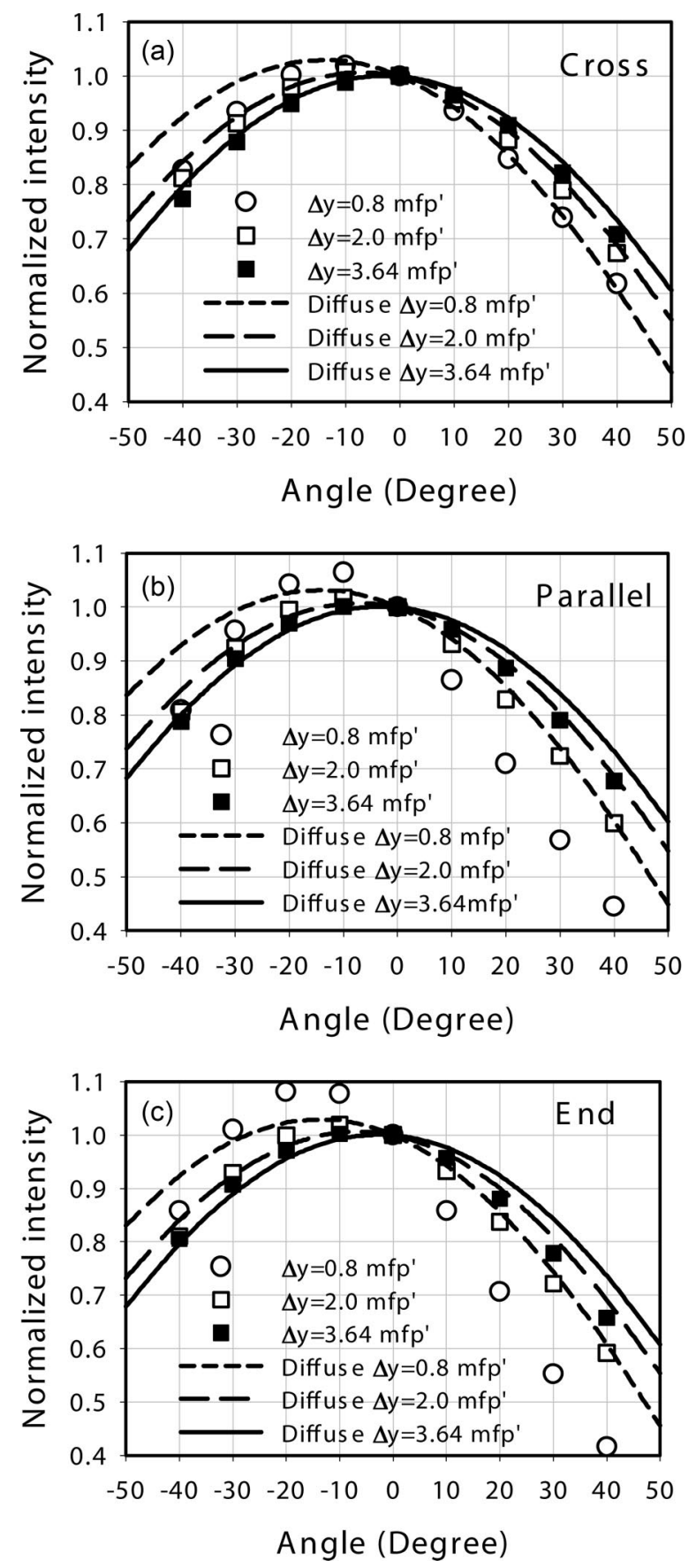

Fig. 7. Normalized angular distribution of diffuse reflectance measured at the incident line in skeletal muscle under $40^{\circ}$ incidence, and with different geometrical arrangements. Optical properties of muscle tissue were: $\mu_{s}{ }^{\prime}=3.6 \mathrm{~cm}^{-1}$ and $\mu_{a}=1.5$ $\times 10^{-2} \mathrm{~cm}^{-1}$ with the cross arrangement, $\mu_{s}{ }^{\prime}=7.1 \mathrm{~cm}^{-1}$ and $\mu_{a}=4.0 \times 10^{-4} \mathrm{~cm}^{-1}$ with the end arrangement, and $\mu_{s}^{\prime}=$ $4.5 \mathrm{~cm}^{-1}$ and $\mu_{a}=6.6 \times 10^{-2} \mathrm{~cm}^{-1}$ with the parallel arrangement.

parallel and end arrangements, respectively. Such different behavior was similar to those observed in the normal incidence scenario.

In all of the samples used in this study, the angular profiles of diffuse reflectance approached the theoretical curve calculated from Eq. (2) if measured far away from the light incident point. At the largest 

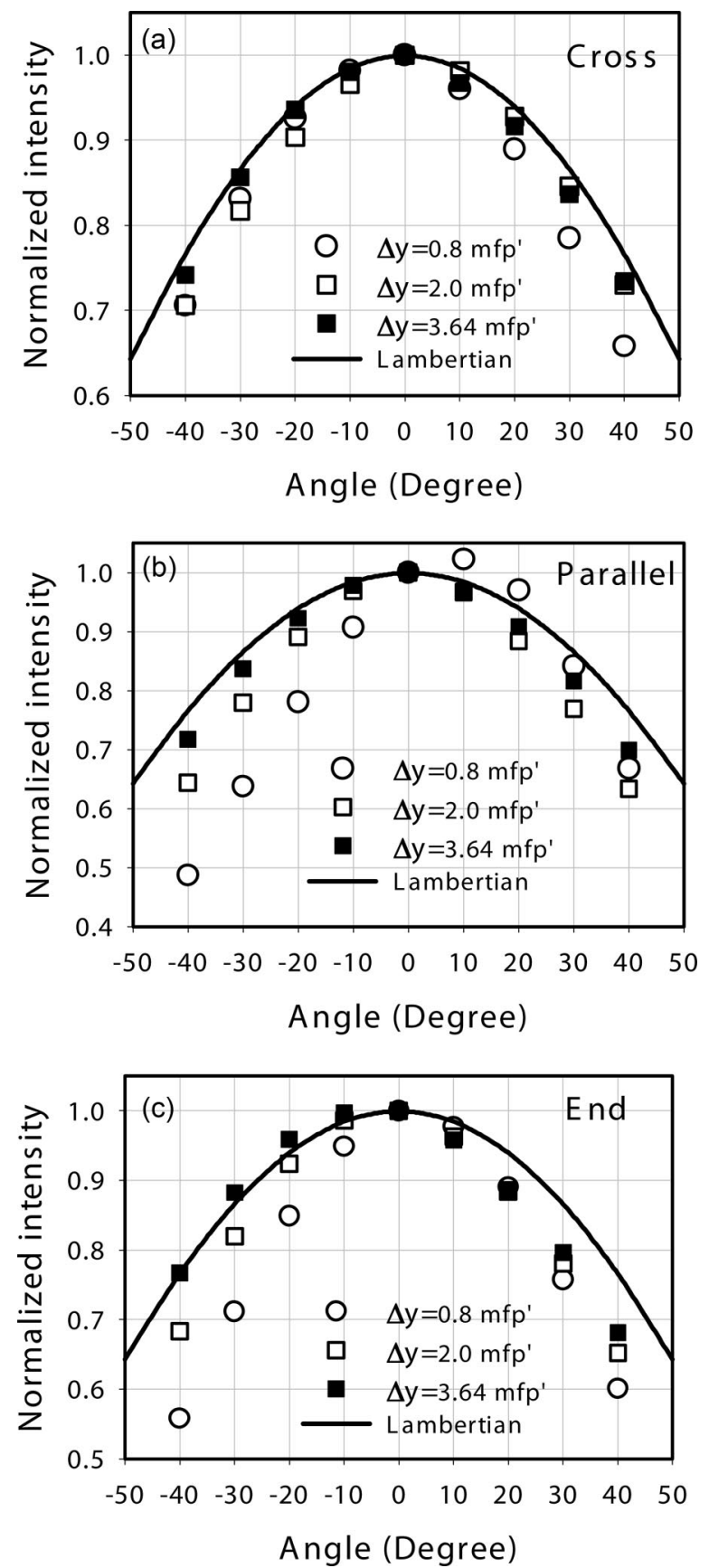

Fig. 8. Normalized angular distribution of diffuse reflectance measured at the diffuse line in skeletal muscle under $40^{\circ}$ incidence, and with different geometrical arrangements. Optical properties of muscle tissue were: $\mu_{s}^{\prime}=3.6 \mathrm{~cm}^{-1}$ and $\mu_{a}=1.5$ $\times 10^{-2} \mathrm{~cm}^{-1}$ with the cross arrangement, $\mu_{s}{ }^{\prime}=7.1 \mathrm{~cm}^{-1}$ and $\mu_{a}=4.0 \times 10^{-4} \mathrm{~cm}^{-1}$ with the end arrangement, and $\mu_{s}{ }^{\prime}=$ $4.5 \mathrm{~cm}^{-1}$ and $\mu_{a}=6.6 \times 10^{-2} \mathrm{~cm}^{-1}$ with the parallel arrangement.

distance $\left(\Delta y=3.64 \mathrm{mfp}^{\prime}\right)$ measured in this study, the relative errors never exceeded $5 \%$ in phantoms, liver tissue, and muscle samples under cross arrangement. Because photons had a greater chance of being diffused at a larger distance, the single scattering characteristic determined by specific anisotropic tissue structures was less significant. Therefore, the mea- sured angular distributions became similar among different tissues.

As observed in a previous study [29], the measured optical properties of muscle samples had significant variations among different measurement configurations. We found that, in this study, the angular profiles of diffuse reflectance were also significantly affected by specific measurement arrangements. Interestingly, when the measurement plane was perpendicular to the skeletal muscle fiber direction, the measured angular distributions were the same as those in the isotropic phantoms in either normal or oblique incidence circumstances.

The aforementioned observation must be originated from detailed mechanisms involved in light-muscle interactions. In skeletal muscles, the periodical sarcomere structures [30] are connected and aligned in the longitudinal direction, which are similar to the regular optical grating. Incident light is preferably diffracted along the muscle fibers. In the dimension that is parallel to muscle fiber direction, the diffraction effects significantly change the scattering phase function and produce a different angular distribution from the isotropic phantom. This effect is significant when the measurement point is close to the incident point, and photons are not fully diffused. On the other hand, the diffraction is not significant in the dimension crossing the fiber direction. Therefore light scattering is similar to the isotropic distributed phantom media. When the muscle fiber end section was used as the measurement surface, the muscle fibers may act as a waveguide and make light transport along fiber direction more easily as indicated in a recent study [14]. With the measurement near the incident point, this directional preference of transport can significantly affect the scattering phase function and cause the measured angular distribution to deviate from the isotropic cases. Nevertheless, further studies are necessary to clarify these effects.

\section{Conclusions}

We compared angular profiles of diffuse reflectance in tissue phantoms, isotropic tissue, and anisotropic tissue. In all of the samples used, the experimental results can be described using the diffuse model when measured far away from the incident point. The diffuse center served as the symmetrical center instead of the incident point. Therefore, when the rotational axis passed through the diffuse center at the sample surface, the obtained angular profiles were symmetrical. On the other hand, a skewed curve appeared when the rotational axis passed through the incident point under an oblique incidence. The results in isotropic liver tissues were the same as in the tissue phantoms. The angular profiles were not sensitive to sample optical properties when the distance measures were in the unit of transport mean-free-path. In skeletal muscles, when measured across the fiber direction, the angular distributions of diffuse reflectance under different conditions were similar to those in isotropic phantoms. These results provide useful information to experimental designs involved with 
diffuse reflectance measurements fiber optic probes. The effects of measurement orientations in skeletal muscle tissues should be considered to achieve a consistent measurement result.

This project was supported in part by National Science Foundation grant CBET-0643190, and the National Research Initiative of the USDA Cooperative State Research, Education, and Extension Service under grant 2006-35503-17619.

\section{References}

1. R. R. Alfano and J. G. Fujimoto, eds., Advances in Optical Imaging and Photon Imaging, Vol. 2 of OSA Trends in Optics and Photonics Series (Optical Society of America, 1996).

2. B. Chance and R. R. Alfano, eds., Optical Tomography and Spectroscopy of Tissue: Theory, Instrumentation, Model, and Human Studies II, Proc. SPIE 2979 (1997).

3. K. Sokolov, M. Follen, and R. Richards-Kortum, "Optical spectroscopy for detection of neoplasia," Curr. Opin. Chem. Biol. 6, 651-658 (2002).

4. I. J. Bigio and S. G. Bown, "Spectroscopic sensing of cancer and cancer therapy: current status of translational research," Cancer Biother. 3, 259-267 (2004).

5. J. Sun, K. Fu, A. Wang, A. W. H. Lin, U. Utzinger, and R. Drezek, "Influence of fiber optic probe geometry on the applicability of inverse models of tissue reflectance spectroscopy: computational models and experimental measurements," Appl. Opt. 45, 8152-8162 (2005).

6. J. Y. Qu, Z. Huang, and J. Hua, "Excitation-and-collection geometry insensitive fluorescence imaging of tissue-simulating turbid media," Appl. Opt. 39, 3344-3356 (2000).

7. S. C. Gebhart, A. Mahadevan-Jansen, and W. C. Lin, "Experimental and simulated angular profiles of fluorescence and diffuse reflectance emission from turbid media," Appl. Opt. 44, 4884-4901 (2005).

8. M. A. ElHelw, B. P. Lo, A. J. Chung, A. Darzi, and G. Z. Yang, "Photorealistic rendering of large tissue deformation for surgical simulation," Lecture Notes Comput. Sci. 3217, 355-362 (2004).

9. A. Kienle, L. Lilge, M. S. Patterson, R. Hibst, R. Steiner, and B. C. Wilson, "Spatially resolved absolute diffuse reflectance measurements for noninvasive determination of the optical scattering and absorption coefficients of biological tissue," Appl. Opt. 35, 2304-2314 (1996).

10. K. M. Hebeda, T. Menovsky, J. F. Beek, J. G. Wolbers, and M. J. C. van Gemert, "Light propagation in the brain depends on nerve fiber orientation," Neurosurgery 35, 720-724 (1994).

11. F. Bevilacqua, P. Marquet, O. Coquoz, and C. Depeursinge, "Role of tissue structure in photon migration through breast tissues," Appl. Opt. 36, 44-51 (1997).

12. S. Nickell, M. Hermann, M. Essenpreis, T. J. Farrell, U. Kramer, and M. S. Patterson, "Anisotropy of light propagation in human skin,” Phys. Med. Biol. 45, 2873-2886 (2000).

13. J. Ranasinghesagara, F. Hsieh, and G. Yao, "A photon migra- tion method for quantifying fiber formation in meat analogs," J. Food Sci. 71, E227-231 (2006).

14. A. Kienle and R. Hibst, "Light guiding in biological tissue due to scattering," Phys. Rev. Lett. 97, 018104 (2006).

15. J. Ranasinghesagara and G. Yao, "Imaging 2D diffuse reflectance in skeleton muscle," Opt. Express 15, 3998-4007 (2007).

16. A. Ishimaru, Wave Propagation and Scattering in Random Media (Academic, 1978), Chaps. 7 and 9.

17. A. J. Welch and M. J. C. Van, Optical-Thermal Response of Laser-Irradiated Tissue (Plenum Press, 1995).

18. S. T. Flock, B. C. Wilson, and M. S. Patterson, "Monte-Carlo modeling of light propagation in highly scattering tissues-II: comparison with measurements in phantoms," IEEE Trans. Biomed. Eng. 36, 1169-1173 (1989).

19. G. Mitic, J. Kolzer, J. Otto, E. Plies, G. Solkner, and W. Zinth, "Time-gated transillumination of biological tissues and tissuelike phantoms," Appl. Opt. 33, 6699-6710 (1994).

20. M. S. Patterson, B. Chance, and B. C. Wilson, "Time resolved reflectance and transmittance for the noninvasive measurement of tissue optical properties," Appl. Opt. 28, 2331-2336 (1989).

21. T. J. Farrell, M. S. Patterson, and B. Wilson, "A diffusion theory model of spatially resolved, steady-state diffuse reflectance for the noninvasive determination of tissue optical properties in vivo," Med. Phys. 19, 879-888 (1992).

22. L.-H. Wang and S. L. Jacques, "Use of a laser beam with an oblique angle of incidence to measure the reduced scattering coefficient of a turbid medium," Appl. Opt. 34, 2362-2366 (1995).

23. S.-P. Lin, L.-H. Wang, S. L. Jacques, and F. K. Tittel, "Measurement of tissue optical properties by the use of obliqueincidence optical fiber reflectometry," Appl. Opt. 36, 136-143 (1997).

24. G. Marquez and L. Wang, "White light oblique incidence reflectometer for measuring absorption and reduced scattering spectra of tissue-like turbid media," Opt. Express 1, 454-460 (1997).

25. H. B. Jiang, G. Marquez, and L. H. Wang, "Particle sizing in concentrated suspensions by use of steady-state, continuouswave photon-migration techniques," Opt. Lett. 23, 394-396 (1998).

26. A. Garcia-Uribe, N. Kehtarnavaz, G. Marquez, V. Prieto, M. Duvic, and L. H. Wang, "Skin cancer detection by spectroscopic oblique-incidence reflectometry: classification and physiological origins," Appl. Opt. 43, 2643-2650 (2004).

27. J. Xia, A. Weaver, D. E. Gerrard, and G. Yao, "Monitoring sarcomere structure changes in whole muscle using diffuse light reflectance," J. Biomed. Opt. 11, 040504 (2006).

28. T. Binzoni, C. Courvoisier, R. Giust, G. Tribillion, T. Gharbi, J. C. Hebden, T. S. Leung, J. Roux, and D. T. Delpy, "Anisotropic photon migration in human skeletal muscle," Phys. Med. Biol. 51, N79-N90 (2006).

29. G. Marquez, L. V. Wang, S. P. Lin, J. A. Schwartz, and S. L. Thomsen, "Anisotropy in the absorption and scattering spectra of chicken breast tissue," Appl. Opt. 28, 2318-2324 (1998).

30. A. M. Pearson and R. B. Young, Muscle and Meat Biochemistry (Academic, 1989). 